\title{
Clinical impact of confinement due to the COVID-19 pandemic on patients with fibromyalgia: A cohort study
}

\section{Javier Rivera ( $\nabla$ javierrivera@ser.es )}

Hospital General Universitario Gregorio Marañón https://orcid.org/0000-0001-8147-5881

Isabel Castrejón

Hospital General Universitario Gregorio Marañón

\section{Laura Vallejo-Slocker}

UNED

\section{Martin Offenbächer}

Gasteiner Heilestollen Hospital

\section{Juan Molina-Collada}

Hospital General Universitario Gregorio Marañón

\section{Laura Trives}

Hospital General Universitario Gregorio Marañón

\section{Katy López}

Hospital General Universitario Gregorio Maranon

\section{Liz Caballero}

Hospital General Universitario Gregorio Maranon

Jameson K. Hirsch

East Tennessee State University

\section{Lauren Toussaint}

Luther College Decorah lowa

\section{Juan C. Nieto}

Hospital General Universitario Gregorio Maranon

Jose M. Alvaro-Gracia

Hospital General Universitario Gregorio Marañón

\section{Miguel A. Vallejo}

UNED

\section{Research article}

Keywords: fibromyalgia, COVID-19, confinement

Posted Date: October 26th, 2020

DOI: https://doi.org/10.21203/rs.3.rs-93711/v1

License: (c) (1) This work is licensed under a Creative Commons Attribution 4.0 International License. Read Full License 
Version of Record: A version of this preprint was published at Clinical and Experimental Rheumatology on June 21st, 2021. See the published version at https://doi.org/10.55563/clinexprheumatol/7lbz8n. 


\section{Abstract}

Background: Data on the effect of the COVID-19 pandemic on fibromyalgia (FM) patients are lacking. FM patients often experience clinical impairment with stress situations.

The objective of this study was to determine whether severity of FM increases because of confinement due to the COVID-19 pandemic.

Methods: Prospective study including patients from the Combined Index of Severity of Fibromyalgia (ICAF) cohort who met the 2010 ACR FM criteria. In this cohort, all patients have a periodical evaluation of their quality of life through two questionnaires, the ICAF -which assesses the ability to perform daily living activities, anxiety and depression-, and through the Patient Global Impression of Change (PGIC), which assesses overall change after a therapeutical intervention. Pre and post-confinement measurements were analyzed. Inferential statistical analysis and ANOVA for repeated measurements were used.

Results: A total of 93 patients received a phone consultation, (95.5\% females), mean (SD) age of 48.23 (8.38) years. Four patients were excluded as presenting COVID-19 and 51 (57\%) completed the post-confinement ICAF. Following confinement, 25 (49\%) patients got worse (group worse) and $26(51 \%)$ patients experienced no change or improved (group stable). Comparisons between pre and post confinement ICAF did not show significant differences in both groups. Passive coping was significantly different in group worse in pre-confinement evaluation. In the $80 \%$ of patients with passive coping predominance there were no change in coping strategy.

Conclusions: No clinical impairment due to COVID-19 confinement occurred. The perceived worsening among FM patients relies primarily on how patients cope with their disease, without a real impact on clinical manifestations.

\section{Background}

In response to the coronavirus pandemic (COVID-19), many European countries have implemented non-pharmaceutical interventions, such as national lockdowns. In Spain, this measure was especially hard, lasting 3 months in which only health and essential public service workers were allowed to move to the workplace.

The psychological impact among the general population caused by quarantine imposed by other infectious diseases has been the subject of a recent review [1]. Most studies show negative psychological effects with symptoms related to post-traumatic stress, confusion and anger. In patients with chronic diseases, this psychological impact may be even higher as these patients suffer added stressors related to their own disease [2]. Patients with fibromyalgia (FM) often have an increase in clinical manifestations of their disease in stressful situations primarily caused by physical or emotional trauma requiring good adaptation mechanisms [3]. In this sense, a stressful situation such as confinement may have increased the severity of clinical manifestations of FM.

The objective of this work was to examine the impact of confinement due to the COVID-19 pandemic in patients with FM.

\section{Methods}

\section{Patients}

In 2010, we started a hospital-based cohort of patients with FM, the Combined Index of Severity in Fibromyalgia (ICAF) cohort, to characterize clinical course and use of treatments through the ICAF questionnaire [4]. Inclusion criteria are: being older than 18 years and fulfilling the 2010 American College of Rheumatology (ACR) FM criteria [5]. At baseline 
main demographic data and information about treatment is collected, and the patients complete the ICAF questionnaire [4]. Treatment is prescribed according to the treating rheumatologist and a follow-up visit is recorded at 3 months in which patients complete a new ICAF with information about treatment. During the follow-up visit, patients also complete a Patient Global Impression of Change (PGIC) [6] questionnaire to evaluate the effect of initial treatment on their overall health status. Subsequently, new evaluations are made at 1, 3 and 5 years of follow-up. Currently, 1100 patients are included in this cohort with different number of visits.

Questionnaires

$\operatorname{ICAF}[4,7]$ is a specific quality-of-life questionnaire for FM patients, offering a total score of the severity of the disease along with four other factors. The physical factor measures the functional ability from the point of view of physical capacity to perform daily activities; the emotional factor measures the severity of clinical manifestations such as anxiety and depression; and two factors of active and passive coping measure the attitude of the patient in coping with their disease. All scores are expressed on a T-scale with an average of 50 and a standard deviation of 10. A high score indicates greater severity, with the exception of the active coping factor that indicates a better coping. The importance of each factor over the total score is different, so the emotional and physical factors are the ones with the highest weight while each coping factor only contributes a small part of the total score severity of the disease [7]. ICAF can also assess the predominance of the type of coping used by patients based on higher scores in active or passive coping. With physical and emotional factors, the predominance of one over the other can also be assessed. This questionnaire has been previously validated in our population [4]. The PGIC [6] is a specific questionnaire to evaluate overall improvement after an intervention. It includes a Likert scale from 1 to 7 ( 1 = much better, 2 = better, $3=$ a little better, $4=$ equal, $5=$ a little worse, 6 = worse or $7=$ much worse) to assess how the patient is feeling after an intervention. This questionnaire has been validated in patients with FM [8] to assess the perception of overall improvement experienced after a therapeutic intervention and is widely used in studies on chronic pain and FM.

Study design

This is a longitudinal study with pre and post-confinement measurements of ICAF and PGIC outcome variables. The confinement period in Spain was from March 14th to June 24th 2020. During this confinement period, all consultations for patients with FM were conducted by telephone at our Unit.

During July 2020, all FM patients included in this cohort and attended by phone consultation on a scheduled basis were asked to assess their status of the severity of symptoms compared to the pre-confinement situation through the PGIC questionnaire (PGIC-post). At the end of the phone consultation, they were mailed an ICAF questionnaire and requested to mail it back (ICAF-post) once completed. All patients verbally consented to the phone consultation. The last available pre-confinement ICAF (ICAF-pre) was used for comparison with the ICAF-post. For comparison purposes, the PGIC after treatment initiation when entering the cohort (PGIC-pre) was used.

\section{Statistical analysis}

For statistical analysis, the t-test was used to compare quantitative variables and the chi-square test for qualitative variables. To analyze the evolution between the ICAF-pre and the ICAF-post, an ANOVA for repeated measurements was used.

\section{Results}

During the study period, 93 FM patients from the cohort treated by phone consultation were identified (Fig. 1). Four patients who had had Covid-19 were excluded, leaving a total of 89 patients for analysis, (95.5\% were females), mean 
(SD) age of $48.2(8.4)$ years.

Only 51 (57.3\%) patients mailed back the ICAF-post questionnaire. No significant differences in age, ICAF-pre, PGIC-pre or PGIC-post were found between patients who mailed the ICAF-post and those who did not.

From the 51 patients with a follow-up evaluation by ICAF-post, the PGIC-post showed improvement in 11 patients (22\%), 25 (49\%) were worse, and 15 (29\%) experienced no change in their clinical status after confinement. For subsequent comparisons two groups were formed: Group worse, including 25 (49\%) patients who had worsened, and Group stable, with 26 (51\%) patients who experienced improvement or no change.

There were not statistically significant differences in age and gender between groups.

In the ICAF-pre, although there was a tendency to a higher severity in patients in the Group worse, this difference did not reach statistically significant differences in each factor except for the passive coping, that was significantly worse in patients in this group $(\mathrm{p}<0.022)$ (Table 1). The ICAF-post analysis showed greater severity in patients in Group worse, with statistically significant differences in total score $(p<0.035)$, physical factor $(p<0.015)$ and passive coping $(p<$ 0.023), but not in emotional factor or active coping (Table 1).

Table 1

Comparison of pre and post-confinement ICAF, total score and individual factors.

\begin{tabular}{|c|c|c|c|c|c|c|c|c|c|c|c|}
\hline \multirow[b]{2}{*}{ Factors } & \multirow[b]{2}{*}{ Group } & \multirow[b]{2}{*}{$\mathrm{n}$} & \multicolumn{2}{|c|}{ ICAF-pre } & \multirow[b]{2}{*}{$\begin{array}{l}\mathrm{p} \\
\text { between } \\
\text { groups }\end{array}$} & \multicolumn{2}{|c|}{ ICAF-post } & \multirow[b]{2}{*}{$\begin{array}{l}\mathrm{p} \\
\text { between } \\
\text { groups }\end{array}$} & \multicolumn{3}{|c|}{$\begin{array}{l}\text { Comparison between } \\
\text { ICAF-pre and ICAF- } \\
\text { post }\end{array}$} \\
\hline & & & mean & SD & & mean & SD & & $\mathrm{F}$ & $d f$ & $\mathrm{p}(\mathrm{es})$ \\
\hline \multirow[t]{2}{*}{ Total } & worse & 25 & 53.75 & 10.96 & & 54.80 & 9.29 & & .291 & 1,24 & $\begin{array}{l}.595 \\
(.012)\end{array}$ \\
\hline & stable & 26 & 50.01 & 13.04 & ns & 47.77 & 13.38 & 0.035 & 1.127 & 1,25 & $\begin{array}{l}.298 \\
(.043)\end{array}$ \\
\hline \multirow[t]{2}{*}{ Physical } & worse & 25 & 54.15 & 11.44 & & 56.77 & 9.10 & & 1.796 & 1,24 & $\begin{array}{l}.193 \\
(.070)\end{array}$ \\
\hline & stable & 26 & 49.99 & 11.26 & ns & 49.16 & 12.23 & 0.015 & .113 & 1,25 & $\begin{array}{l}.740 \\
(.004)\end{array}$ \\
\hline \multirow[t]{2}{*}{ Emotional } & worse & 25 & 52.55 & 10.93 & & 53.58 & 9.10 & & .277 & 1,24 & $\begin{array}{l}.604 \\
(.011)\end{array}$ \\
\hline & stable & 26 & 50.81 & 12.85 & ns & 48.28 & 12.78 & ns & 1.696 & 1,25 & $\begin{array}{l}.205 \\
(.064)\end{array}$ \\
\hline \multirow[t]{2}{*}{$\begin{array}{l}\text { Active } \\
\text { coping }\end{array}$} & worse & 25 & 50.10 & 9.20 & & 52.20 & 10.13 & & 1.503 & 1,24 & $\begin{array}{l}.232 \\
(.059)\end{array}$ \\
\hline & stable & 26 & 51.95 & 12.54 & ns & 54.11 & 10.65 & ns & 2.689 & 1,25 & $\begin{array}{l}.114 \\
(.097)\end{array}$ \\
\hline \multirow[t]{2}{*}{$\begin{array}{l}\text { Passive } \\
\text { coping }\end{array}$} & worse & 25 & 55.71 & 7.97 & & 58.10 & 9.58 & & 1.738 & 1,24 & $\begin{array}{l}.200 \\
(.068)\end{array}$ \\
\hline & stable & 26 & 50.22 & 8.51 & 0.022 & 51.56 & 10.23 & 0.023 & .407 & 1,25 & $\begin{array}{l}.529 \\
(.016)\end{array}$ \\
\hline
\end{tabular}


Abbreviation. ICAF: Combined Index of Severity of Fibromyalgia, n: number of patients, SD: standard deviation, F: value, df: degree of freedom, p: value, es: effect size.

When analyzing the evolution of patient severity comparing ICAF-pre with ICAF-post, no significant differences were found in either group suggesting that there had been no clinical impairment in patients with respect to their previous situation (Table 1).

The predominance of the factors in the ICAF-pre (passive $v s$ active and emotional $v s$ physical) in both groups is shown in Table 2.

Table 2

Predominance of factors in ICAF-pre.

\begin{tabular}{|llll|}
\hline Predominance & Patients & Group & Group \\
& $\mathbf{n}(\%)$ & worse & stable \\
& & $\mathbf{n}(\%)$ & $\mathbf{n}(\%)$ \\
\hline Passive coping & $24(47 \%)$ & $15(60 \%)$ & $9(35 \%)$ \\
\hline Emotional factor & $26(51 \%)$ & $12(48 \%)$ & $14(54 \%)$ \\
\hline
\end{tabular}

Abbreviation. n: number of patients

Predominance of passive coping was higher in Group worse, but it did not reach statistical significance. The predominance of emotional factors was also similar in both groups. Of the 15 patients in Group worse with the predominance of passive coping in the ICAF-pre, 12 (80\%) of them continued to have a predominance of passive coping when they performed the ICAF-post. In the PGIC-pre, of the total number of patients, 17 (33\%) patients responded to being worse after the initial treatment of the first visit. Of these, 11 (65\%) patients responded again that they were worse in the PGIC-post.

\section{Discussion}

Patients with FM experienced a worsening in their health status after confinement during the COVID-19 pandemic in our cohort. Because we initially excluded all patients who had had clinical manifestations compatible with COVID-19, the worsening of their health status may be attributed directly to the stressors surrounding the confinement.

To our knowledge, this is the first study specifically designed to analyze the impact of COVID-19 pandemic quarantine on the health status in patients with FM. In a recent study [1] evaluating the impact of confinement on patients with various chronic diseases, the authors found that $48 \%$ of them had worsened their perceived health status. Our findings show that the impact of confinement on FM patients is probably similar to that of any other chronic disease.

In patients who reported a worsening of their health status after confinement, the total score of ICAF, as well as the physical factor (which measures variables such as pain, sleep quality or daily life activities), and the emotional factor (which mainly measures anxiety and depression), showed no significant differences with the situation before confinement, suggesting that there had not been a clear worsening of the main clinical manifestations of FM. Only passive coping (which measures the patient's attitude towards their disease along with the coping strategies they are able to develop) was significantly worse both in the previous situation and after confinement. In this sense, $80 \%$ of the patients who were worse had already had a predominant passive coping before confinement. 
It is noteworthy that passive coping has little influence on the total score of the ICAF questionnaire explaining only $6 \%$ of the variance in the construction of the questionnaire, with the physical and emotional factors being the most relevant [7]. This suggests that the perceived worsening among FM patients in our study as a result of COVID-19 pandemic confinement depends primarily on how patients cope with their disease, without a real impairment of the clinical manifestations measured by physical or emotional factors. In the general population, some variables such as female sex, the absence of higher education, the number of people at home or lonely, are associated with a worse situation after confinement [1]. On the other hand, in patients with chronic diseases the variables associated with worsening are also related to aspects of daily life and do not seem to be related to the clinical manifestations of the disease, except for the duration of the disease itself [2]. In other situations of collective stress in society, such as the World Trade Center terrorist attack in 2001, there was also no significant worsening of symptoms in FM patients [9]. In this study, previous depressive pathology was also unable to predict worsening of symptoms, as well as previous FM symptoms such as pain or other psychiatric symptoms [9].

In addition to the psychological impact of confinement, as we have studied here, there are other possibilities for the COVID-19 pandemic to produce an impairment in the health situation of FM patients. Currently it is not known whether the infection with corononavirus SARS-cov2 may produce long-term chronic clinical manifestations such as fatigue, myalgias, arthralgias or cognitive alterations compatible with chronic fatigue syndrome or FM, as we know to occur in other infectious viral diseases [10].

If the current COVID-19 pandemic lasts much longer, as it seems to be happening, an increase in new FM cases may occur, especially among the most predisposed patients or those with other chronic inflammatory disease that adds another major stressor for these patients.

The main strength of our study is that we have a measure of quality of life in FM patients prior to the confinement by the COVID-19 pandemic that has allowed us to compare with the situation after the confinement. Several limitations are present in our study. First, the low response rate of 57\%, which may underestimate the impact of confinement on FM. Second, we did not collect other relevant variables such as level of education, the number of people at home or lonely, which have been associated with a worse situation after confinement in the general population. Third, our results need to be evaluated in other cohorts of FM patients.

\section{Conclusions}

In this study, we show that confinement during the COVID-19 pandemic has a negative impact on patients with FM. This depends primarily on how patients cope with their disease, without a real impairment of clinical manifestations.

\section{Abbreviations}

ACR American College of Rheumatology

ANOVA Analysis of varianza

COVID-19 Coronavirus disease 2019

FM Fibromyalgia

ICAF Combined Index of Severity of Fibromyalgia

PGIC Patient Global Impression of Change

Page $7 / 10$ 


\section{Declarations}

\section{Ethics approval and consent to participate}

The present study was approved by the ethics committee of Hospital General Universitario Gregorio Marañón. Instituto de Investigación Sanitaria Gregorio Marañón (IiSGM). Madrid. Spain.

All the patients gave their verbal consent to participate in the study.

\section{Consent for publication}

Not Applicable.

\section{Availability of data and materials}

The datasets used and/or analysed during the current study are available from the corresponding author on reasonable request.

\section{Competing interests}

All the authors declare that they have no competing interests.

\section{Funding}

This study was funded by the Ministerio de Economía y Competitividad (MINECO). Proyectos I+D+I (Retos), Spanish Government under the Grant PSI2017-85447-R.

\section{Authors' contributions}

RJ designed the study with input from VMA, A-GJM and CI. V-SL, RJ and VMA performed analysis of data. RJ wrote the manuscript with inputs from OM, M-CJ, TL, LK, CL, HJK, NJC and TL.

\section{Acknowledgements}

Not Applicable.

\section{Authors' information}

${ }^{1}$ Servicio de Reumatología. Hospital General Universitario Gregorio Marañón. Instituto de Investigación Sanitaria Gregorio Marañón (liSGM). Madrid. Spain.

${ }^{2}$ Department of Clinical Psychology, National Distance Education University (UNED), Madrid, Spain.

${ }^{3}$ Gasteiner Heilstollen Hospital, Bad Gastein-Böckstein, Austria.

${ }^{4}$ Department of Psychology, East Tennessee State University, Johnson City, USA

${ }^{5}$ Department of Psychology, Luther College, Decorah, lowa, USA.

\section{References}


1. Brooks S, Webster R, Smith L, Woodland L, Wessely S, Greenberg N, Rubin G. The psychological impact of quarantine and how to reduce it: Rapid review of the evidence. Lancet. 2020;395:912-20.

2. Elran-Barak R, Mozeikov M. One Month into the Reinforcement of Social Distancing due to the COVID-19 Outbreak: Subjective Health, Health Behaviors, and Loneliness among People with Chronic Medical Conditions. Int J Environ Res Public Health. 2020;17(15):5403.

3. Yavne Y, Amital D, Watad A, Tiosano S, Amital H. A systematic review of precipitating physical and psychological traumatic events in the development of fibromyalgia. Semin Arthritis Rheum. 2018;48:121-33.

4. Vallejo MA, Rivera J, Esteve-Vives J, Rejas J, Group ICAF. A confirmatory study of the Combined Index of Severity of Fibromyalgia (ICAF*): factorial structure, reliability and sensitivity to change. Health Qual Life Outcomes. 2011;9:39.

5. Wolfe F, Clauw DJ, Fitzcharles MA, Goldenberg DL, Katz RS, Mease P, Russell AS, Russell IJ, Winfield JB, Yunus MB. The American College of Rheumatology preliminary diagnostic criteria for fibromyalgia and measurement of symptom severity. Arthritis Care Res (Hoboken). 2010;62(5):600-10.

6. Guy W. ECDEU Assessment Manual for Psychopharmacology. Washington, DC, USA: Government Printing Office; 1976.

7. Vallejo MA, Rivera J, Esteve-Vives J, Group ICAF. Development of a self-reporting tool to obtain a combined index of severity of fibromyalgia (ICAF). Health Qual Life Outcomes. 2010;8:2.

8. Rampakakis E, Ste-Marie PA, Sampalis JS, Karellis A, Shir Y, Fitzcharles MA. Real-life assessment of the validity of patient global impression of change in fibromyalgia [published correction appears in RMD Open.

2015;1(1):e000146corr1]. RMD Open. 2015;1(1):e000146.

9. Raphael KG, Natelson BH, Janal MN, Nayak S. A community-based survey of fibromyalgia-like pain complaints following the World Trade Center terrorist attacks. Pain. 2002;100(1-2):131-39.

10. Clauw DJ, Häuser W, Cohen SP, Fitzcharles MA. Considering the potential for an increase in chronic pain after the COVID-19 pandemic [published online ahead of print, 2020 Jun 26]. Pain.

2020;10.1097/j.pain.0000000000001950.

\section{Figures}




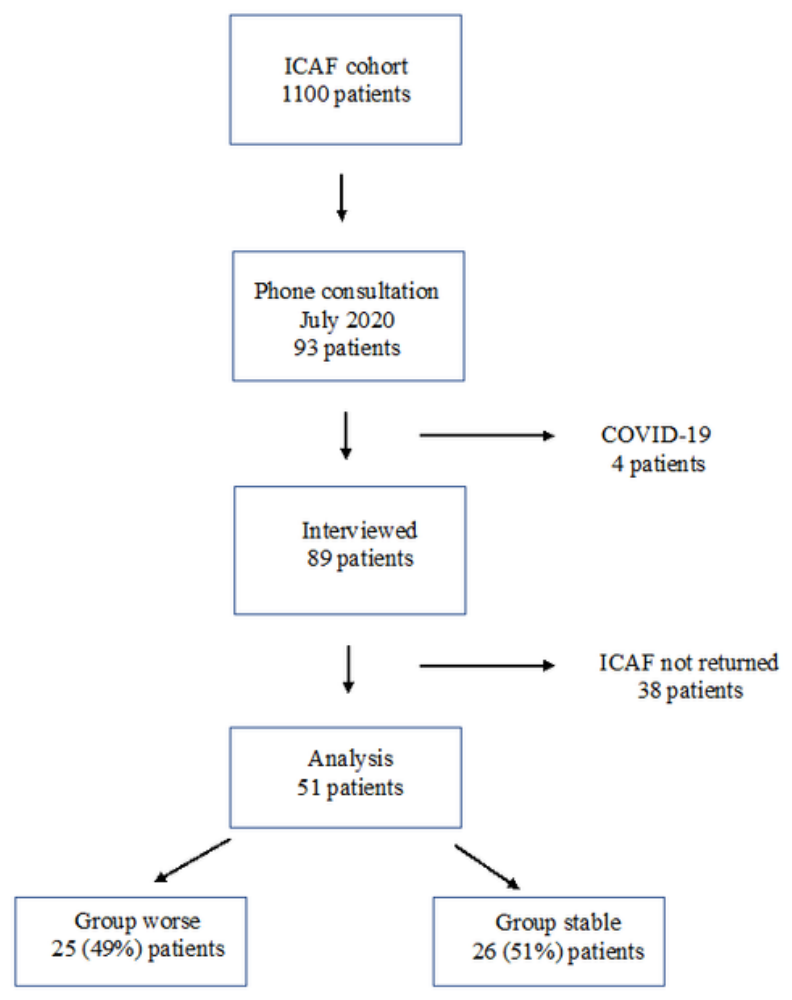

Figure 1

Patients selection flow-chart. 